\title{
Quantitative rigidity evaluation during deep brain stimulation surgery - a preliminary study
}

S. Hemm-Ode, Institute for Medical and Analytical Technologies, University of Applied Sciences Northwestern Switzerland, Muttenz, Switzerland, simone.hemm@fhnw.ch

D. Gmünder, Institute for Medical and Analytical Technologies, University of Applied Sciences Northwestern Switzerland, Muttenz, Switzerland, Dagmar.gmuender@gmail.com

A. Shah, Institute for Medical and Analytical Technologies, University of Applied Sciences Northwestern Switzerland, Muttenz, Switzerland, ashesh.shah@fhnw.ch

M. Ulla, Neuro-Psycho-pharmacologie des Systèmes dopaminergiques sous-corticaux, Université d'Auvergne ; Service de Neurologie, CHU de Clermont-Ferrand, Clermont-Ferrand, France, mulla@chu-clermontferrand.fr

J. J. Lemaire, Image-Guided Clinical Neuroscience and Connectomics - ISIT, Université d'Auvergne ; Service de Neurochirurgie, CHU de Clermont-Ferrand, Clermont-Ferrand, France, jilemaire@chu-clermontferrand.fr

J. Coste, Image-Guided Clinical Neuroscience and Connectomics - ISIT, Université d'Auvergne ; Service de Neurochirurgie, CHU de Clermont-Ferrand, Clermont-Ferrand, France, jcoste@chu-clermontferrand.fr

\section{Introduction}

Deep brain stimulation (DBS) is a common neurosurgical procedure for relieving movement related disorders such as Parkinson's disease. DBS extends uncertainties associated with suboptimal target selection. Our aim was to evaluate the feasibility to objectively assess clinical effects obtained during intraoperative test stimulation based on acceleration measurements of the neurologist's wrist.

\section{Methods}

One patient referred for bilateral DBS-implantation for the treatment of Parkinson's disease was included in the study. A 3-axis accelerometer was fixed on the neurologist's wrist during intraoperative test stimulation. While the intensity of electric current used for stimulation was increased, the neurologist continuously moved the patient's wrist to determine the moment of and the amplitude at rigidity release ("stimulation threshold"). For each test stimulation position, differrent mathematical features were determined and statistically compared a) for the time period before reaching the stimulation threshold that had been identified by the neurologist and b) after reaching the threshold. We then visually identified the stimulation thresholds that would have been chosen based on the acceleration signal alone and compared them to the ones subjectively identified by the neurologist.

\section{Results}

A statistical significant change in rigidity $(\mathrm{p}<0.01)$ could be identified for signal entropy, energy and standard deviation. The signal energy seemed to be the most sensible parameter showing a higher percentage change compared to the initial clinical state. The stimulation threshold identified based on the acceleration signal was in most cases lower than the subjectively determined one.

\section{Conclusion}

The present study has demonstrated the feasibility to perform rigidity assessments from the acceleration signal of the wrist. The stimulation threshold was confirmed by the acceleration measurements, and it seems that the measurements were more sensitive than the neurologist's evaluation. Results have to be confirmed by a larger clinical study. 